This paper is a non peer-reviewed preprint submitted to EarthArXiv. Future version of this paper may contain slightly different content. Please feel free to contact the authors; your feedback is welcome and appreciated. 


\title{
LaserTRAM-DB: A Time Resolved Analysis Module for the complete reduction of Laser Ablation Inductively Coupled Plasma Mass Spectrometry data
}

\author{
Jordan Lubbers*, Adam Kent, Chris Russo \\ W.M. Keck Collaboratory for Plasma Spectrometry \\ College of Earth, Ocean, and Atmospheric Sciences, Oregon State University \\ *jelubber@gmail.com
}

\begin{abstract}
Summary
LaserTRAM-DB is a dashboard for the complete processing pipeline of Laser Ablation Inductively Coupled Plasma Mass Spectrometry (LA-ICP-MS) data in complex materials such as geologic samples. As LA-ICPMS data in geologic samples frequently have multiple phases, inclusions, and other compositional complexities within them that do not represent the material of interest, user interaction is required to filter unwanted signals out of the overall ablation signal. LaserTRAM-DB allows the user to filter which portion of the ablation peak is utilized in calculating concentrations, subsequently allowing for more accurate data to be obtained. Furthermore, it allows for the processing of both individual spot analysis data and a line of spots gathered in rapid succession, reducing the time required for data reduction while preserving spatial definition and still ensuring data quality.
\end{abstract}

\section{Statement of Need}

With a wide array of applications in the natural sciences (e.g., Fritz-Endres and Fehrenbacher, 2021; Caricchi et al., 2020; Loewen and Kent, 2012; Lukács et al., 2021), laser ablation inductively coupled plasma mass spectrometry (LA-ICP-MS) is a now a commonplace tool for the gathering of in situ trace element (i.e., < $0.1 \mathrm{wt} \%$ ) data from solid materials. The last two decades have seen significant advances in both instrument capabilities and operating software, allowing users to generate large volumes of in situ geochemical data in comparatively little time to previous methodologies (i.e., micro-drilling) while still maintaining high degrees of accuracy and precision.

Raw data output from LA-ICP-MS, however, is in the form of counts per second (cps) for the selected analyte isotopes, not elemental concentrations. In order to be converted into accurate concentrations, a modest amount of user input and interpretation is required and should not be automated. Currently, there are several proprietary and open-source softwares for LA-ICP-MS data reduction to accomplish this task: SILLS - Guillong et al. (2008); Iolite - Paton et al. (2011); LAtools - Branson et al. (2019); Termite - Mischel et al. (2017); GLITTER - Macquarie University GEMOC; and countless other "in house" spreadsheet-based tools. All have their strengths and weaknesses, however, there is yet to be a powerful, web-hosted Graphical User Interface (GUI) e.g. Figure 1 . Built primarily using Plotly-Dash [Plotly Technologies Inc.], numpy (Harris et al., 2020), and pandas (McKinney et al., 2010), we present a completely open-source dashboard: Laser Time Resolved Analysis Module Dashboard (LaserTRAM-DB) that allows the user to calculate concentrations from raw LA-ICP-MS data with the flexibility of a GUI interface while maintaining the performance of the numerical python ecosystem. Furthermore, by simultaneously displaying both raw data cps and internal standard normalized cps, it allows for rapid decision making about data quality to be determined. It is comprised of three parts:

1. LaserTRAM: Choosing an interval of interest from raw cps data in individual spot analyses and normalizing it to an internal standard. 
2. LaserTRAM profiler: Functionally the same as LaserTRAM, however has tools that allow for the rapid inspection of a line of spot analyses gathered in quick succession.

3. LaserCalc: Takes the output from either LaserTRAM or LaserTRAM profiler and converts the normalized data into concentrations using the equations outlined below.

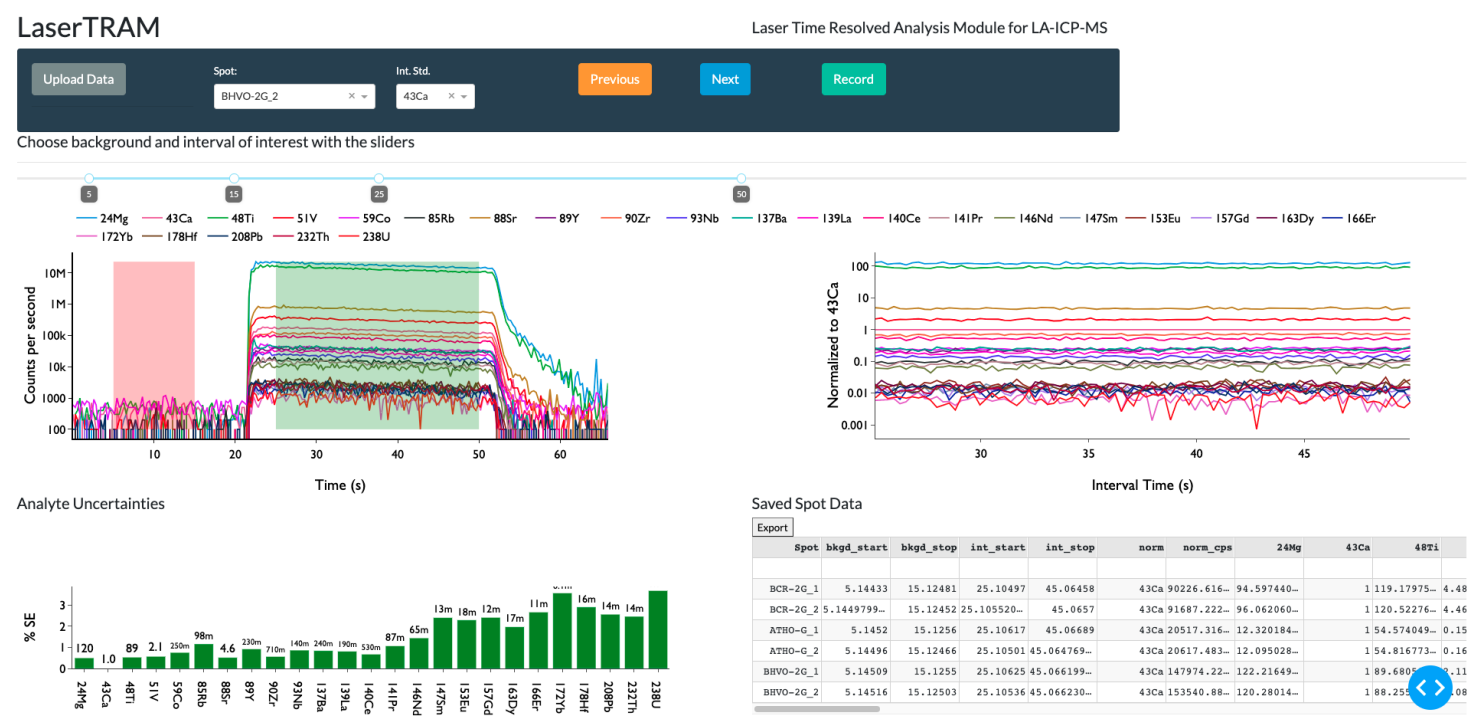

Figure 1: Example of the LaserTRAM-DB interface showing its capability to filter portions of spectra for a given spot analysis based on user input. Shown is a spot analysis from standard reference material BHVO-2G.

\section{Governing Equations}

We calculate the concentration of analyte $(i)$ in an unknown material $(u)$ using the following relationship from Longerich et al. (1996):

$$
C_{i}{ }^{u}=\frac{R_{i}{ }^{u}}{S}
$$

Where $C_{i}{ }^{u}$ and $R_{i}{ }^{u}$ are the concentration of analyte and count rate of analyte $(i)$ in the unknown material, respectively, and $S$ is the normalized sensitivity. When using naturally occuring internal standards, $S$ can be defined as:

$$
S=\frac{R_{i}{ }^{s t d}}{{C_{i}}^{s t d}}\left[\frac{R_{n}{ }^{u}}{{R_{n}}^{s t d}} \frac{C_{n}{ }^{s t d}}{C_{n}{ }^{u}}\right]
$$

$R_{i}{ }^{s t d}$ and $C_{i}{ }^{s t d}$ are the count rate and and concentration of analyte $(i)$ in the calibration standard, $R_{n}{ }^{u}$ and $R_{n}{ }^{s t d}$ are the mean count rates of the internal standard in the unknown material and calibration standard, $C_{n}{ }^{u}$ and $C_{n}{ }^{\text {std }}$ are the concentrations of the internal standard in the unknown material and calibration standard.

Kent and Ungerer (2006) re-arrange this relationship such that the count rate expressions always contain unknown analytes in the numerator:

$$
C_{i}{ }^{u}=C_{n}{ }^{u} \frac{\left[\frac{C_{i}{ }^{s t d}}{C_{n}{ }^{s t d}}\right]}{\left[\frac{R_{i}{ }^{\text {std }}}{R_{n}{ }^{s t d}}\right]} \frac{R_{i}{ }^{u}}{R_{n}{ }^{u}}
$$




\section{Determining Normalized Ratios}

The purpose of LaserTRAM-DB is to give the user complete control over which portion of the analytical spectra gets used in calculating concentrations (e.g., filtering out portions of the signal not reflective of the material under investigation). In complex natural materials, selection of this interval and an overall judgement about data quality require an operator to make a decision. This sofware is optimized to allow that decision to be made as efficiently and rapidly as possible.

When a given interval from the analytical spectra has been chosen, every analyte is normalized to a chosen internal standard. LaserTRAM-DB allows for any analyte in the experiment to be used as the internal standard. Prior to normalization to an internal standard, raw data first has the background analyte levels subtracted from it. Background is determined by taking the median counts per second value for each analyte over the specified background range. Once data have been background subtracted, each normalized ratio is calculated the following way:

$$
N_{i}=\text { median }\left[\frac{c p s_{i}}{c p s_{i s}}\right]
$$

Where $c p s_{i}$ is the background subtracted counts per second data for analyte $(i)$, and $c p s_{i s}$ is the background subtracted counts per second data for the internal standard. Since counts per second is analogous to count rate above in Equation 3, we can simplify the above relationship to now reflect our $N_{i}$ values:

$$
C_{i}{ }^{u}=C_{n}{ }^{u} \frac{\left[\frac{C_{i}^{s t d}}{C_{n}{ }^{s t d}}\right]}{N_{i}{ }^{s t d}} N_{i}{ }^{u}
$$

Here, $N_{i}{ }^{s t d}$ and $N_{i}{ }^{u}$ are the normalized counts per second value of analyte $i$ in the calibration standard and unknown, respectively. The uncertainty for any given normalized ratio is expressed as:

$$
S E=\frac{\sigma_{N_{i}}}{\sqrt{n}}
$$

$\sigma_{N}$ is the standard deviation of a given analyte's normalized ratio for the interval and $n$ is the number of time steps in the interval (i.e., cycles through the mass range). The relative standard error is then:

$$
R S E_{i}{ }^{u}=\left[\frac{S E}{N_{i}}\right] 100
$$

Detection limits for each analyte are 3 standard deviations above the mean of the background levels as defined earlier. This reflects $99.7 \%$ confidence that the analyte is above background levels. This is standard practice in LA-ICP-MS data reduction. To reflect this in data output, normalized ratios below detection limit are coded to show up as negative ratios in LaserTRAM that then get turned into "b.d.l." values in LaserCalc.

\section{Concentrations of internal standard in unknown}

To calculate concentrations of a given analyte list in an unknown sample, the concentration of the internal standard must be known. LaserCalc takes these concentrations in the form of wt\% oxide and utilizes user interaction to input concentrations of the internal standard and its relative uncertainty. A default value of $1 \%$ is used for this, but may be updated by the user.

\section{Drift Correction}

To check for drift in calibration standard normalized ratios over time, a linear regression is applied to the calibration standard for each analyte, where the dependent variable is the count rate normalized to the internal standard and the independent variable is the analysis number (Figure 2).

This regression and the observed data then receive a Root Mean Squared Error (RMSE) value. A linear drift correction is applied to an analyte if the relative RMSE value for a given analyte is less than the RSE. Here RSE is defined as: 


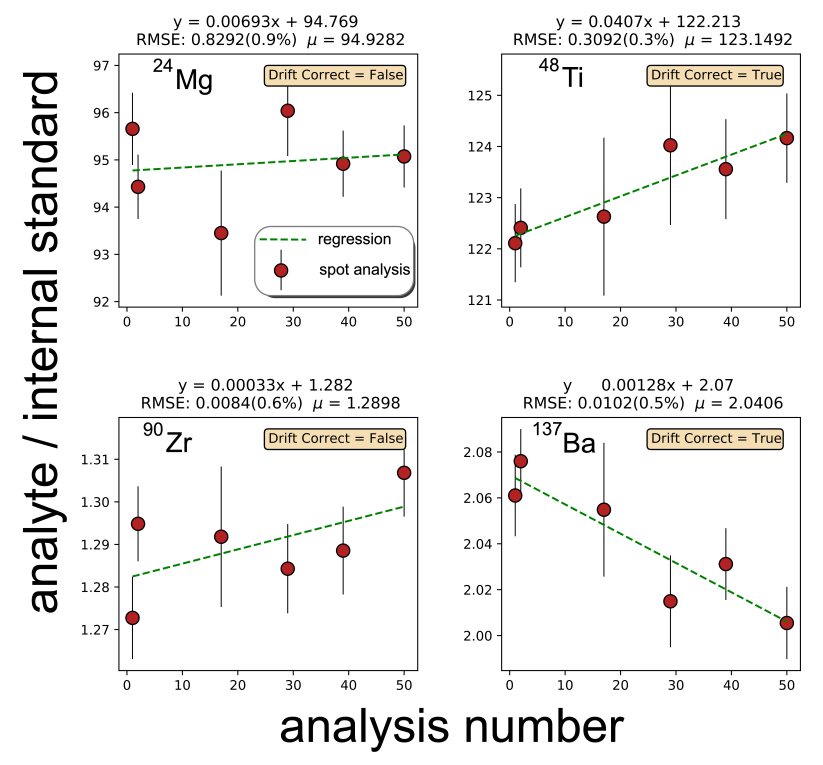

Figure 2: Drift correction test for selected analytes in Figure 1 illustrating analytes that are both drift corrected and not drift corrected in LaserCalc.

$$
R S E_{i}{ }^{s t d}=\left[\frac{\frac{\sigma_{i}}{\sqrt{n_{i}}}}{\mu_{i}}\right] 100
$$

Where $\sigma_{i}$ and $\mu_{i}$ are the standard deviation and mean of all of the calibration standard normalized ratios respectively and $n_{i}$ is the total number of calibration standard analyses for analyte $(i)$.

In brief, the only way drift correction happens is if there is a sufficiently large linear change in normalized count rates for the calibration standard over time that causes the RMSE of the regression to have lower values than the standard error of the mean. This drift correction then uses the regression parameters (e.g., slope and intercept) to calculate a normalized count rate for the calibration standard at the point in time where an unknown was analyzed:

$$
C_{i}^{u}=C_{n}^{u} \frac{\left[\frac{C_{i}{ }^{s t d}}{C_{n}{ }^{s t d}}\right]}{\left[m_{i} x+b_{i}\right]} N_{i}^{u}
$$

Where $m$ is the regression slope, $x$ is the analysis number, and $b$ is the intercept for analyte $i$.

\section{Uncertainties}

Calculating concentrations of a given analyte in an unknown material can be considered a series of nested quotients and products. Therefore, we quantify the overall uncertainty of a given analyte as Taylor (1997) p.61:

$$
\sigma_{C_{i}}=C_{i}{ }^{u} \sqrt{\left(\frac{\sigma_{C_{u}}{ }^{n}}{C_{u}{ }^{n}}\right)^{2}+\left(\frac{\sigma_{C_{i} s t d}}{C_{i}{ }^{s t d}}\right)^{2}+\left(\frac{\sigma_{C_{n} s t d}}{C_{n}{ }^{s t d}}\right)^{2}+\left(R S E_{i}{ }^{s t d}\right)^{2}+\left(R S E_{i}{ }^{u}\right)^{2}}
$$

For analytes where drift correction has been applied, $R S E_{i}{ }^{s t d}$ is replaced with:

$$
100\left[\frac{R M S E_{i}}{\mu_{i}}\right]
$$

Where $R M S E_{i}$ is the Root Mean Squared Error as specified in the Drift Correction section. 


\section{Data Output}

Both steps of the data processing pipeline (e.g., LaserTRAM and LaserCalc) allow the user to output progress in the form of an Excel spreadsheet that mimics the application datatable hosted in the web browser. Output follows tidy data format (e.g., Wickham, 2014) where columns contain observation attributes (i.e., analytes values and associated metadata) and rows denote observations (i.e., an individual spot analysis).

\section{Installation and Use}

The easiest way to use LaserTRAM-DB is to use the following link:

- https://lasertram-db.herokuapp.com/

Alternatively, LaserTRAM-DB can be installed locally and run by creating a virtual environment. If you are new to python, we recommend doing this through Anaconda.



When the program is running, copy and paste the provided link provided in the terminal window into the browser window and the app will run. From now on any time you wish to use the program, simply re-activate the virtual environment and run the script like above:

conda activate lasertram-db

$\mathrm{cd} /$ path/to/laserTRAM-DB

python lasertram-db.py

\section{Caveats}

On windows you may need to add the following channel for downloading the requirements.txt file:

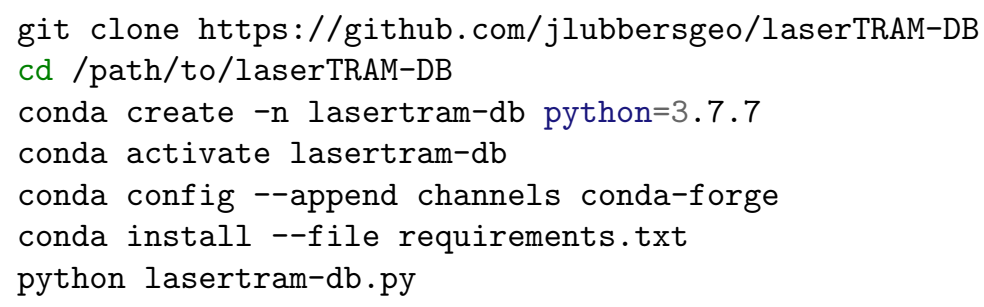

\section{Demos}

Video tutorials on how to use each piece of software can be found at the following links:

- LaserTRAM video

- LaserTRAM profiler video

- LaserCalc video 


\section{Citing}

If you use this software for your research, please cite it as follows:

- In text: Lubbers et al. (2021)

- bibtex:

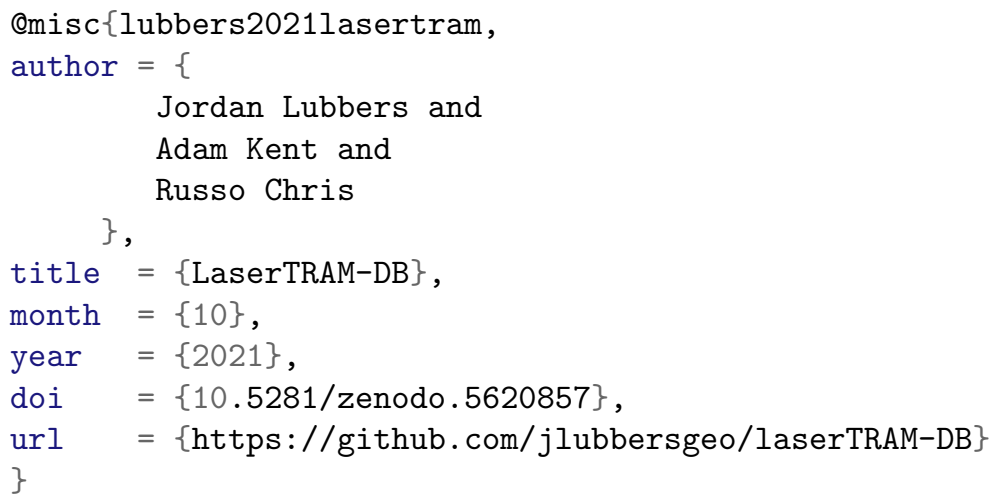

\section{Acknowledgements}

We express our gratitude to the W.M. Keck Foundation for helping foster the labarotory environment that led to the genesis of this software as well as NSF project grants 1763639, 1948862, and 1654275 .

\section{References}

O. Branson, J. S. Fehrenbacher, L. Vetter, A. Y. Sadekov, S. M. Eggins, and H. J. Spero. Latools: A data analysis package for the reproducible reduction of la-icpms data. Chemical Geology, 504:83-95, 2019. doi: 10.1016/j.chemgeo.2018.10.029.

L. Caricchi, M. Petrelli, E. Bali, T. Sheldrake, L. Pioli, and G. Simpson. A data driven approach to investigate the chemical variability of clinopyroxenes from the 2014-2015 holuhraun-bárdarbunga eruption (iceland). Frontiers in Earth Science, 8:18, 2020. doi: 10.3389/feart.2020.00018.

T. Fritz-Endres and J. Fehrenbacher. Preferential loss of high trace element bearing inner calcite in foraminifera during physical and chemical cleaning. Geochemistry, Geophysics, Geosystems, 22(1): e2020GC009419, 2021. doi: 10.1029/2020gc009419.

M. Guillong, D. L. Meier, M. M. Allan, C. A. Heinrich, and B. W. Yardley. Appendix a6: Sills: A matlabbased program for the reduction of laser ablation icp-ms data of homogeneous materials and inclusions. Mineralogical Association of Canada Short Course, 40:328-333, 2008.

C. R. Harris, K. J. Millman, S. J. van der Walt, R. Gommers, P. Virtanen, D. Cournapeau, E. Wieser, J. Taylor, S. Berg, N. J. Smith, et al. Array programming with numpy. Nature, 585(7825):357-362, 2020. doi: $10.1038 / \mathrm{s} 41586-020-2649-2$.

A. J. Kent and C. Ungerer. Analysis of light lithophile elements (li, be, b) by laser ablation icp-ms: Comparison between magnetic sector and quadrupole icp-ms. American Mineralogist, 91(8-9):1401-1411, 2006. doi: $10.2138 /$ am.2006.2030.

M. W. Loewen and A. J. Kent. Sources of elemental fractionation and uncertainty during the analysis of semi-volatile metals in silicate glasses using la-icp-ms. Journal of Analytical Atomic Spectrometry, 27(9): 1502-1508, 2012. doi: 10.1039/c2ja30075c. 
H. P. Longerich, S. E. Jackson, and D. Günther. Inter-laboratory note. laser ablation inductively coupled plasma mass spectrometric transient signal data acquisition and analyte concentration calculation. Journal of analytical atomic spectrometry, 11(9):899-904, 1996. doi: 10.1039/ja9961100899.

J. Lubbers, A. Kent, and R. Chris. Lasertram-db, 10 2021. URL https://github.com/jlubbersgeo/ laserTRAM-DB.

R. Lukács, L. Caricchi, A. K. Schmitt, O. Bachmann, O. Karakas, M. Guillong, K. Molnár, I. Seghedi, and S. Harangi. Zircon geochronology suggests a long-living and active magmatic system beneath the ciomadul volcanic dome field (eastern-central europe). Earth and Planetary Science Letters, 565:116965, 2021. doi: 10.1016/j.epsl.2021.116965.

W. McKinney et al. Data structures for statistical computing in python. In Proceedings of the 9th Python in Science Conference, volume 445, pages 51-56. Austin, TX, 2010. doi: 10.25080/majora-92bf1922-00a.

S. A. Mischel, R. Mertz-Kraus, K. P. Jochum, and D. Scholz. Termite: An r script for fast reduction of laser ablation inductively coupled plasma mass spectrometry data and its application to trace element measurements. Rapid Communications in Mass Spectrometry, 31(13):1079-1087, 2017. doi: 10.1002/rcm. 7895 .

C. Paton, J. Hellstrom, B. Paul, J. Woodhead, and J. Hergt. Iolite: Freeware for the visualisation and processing of mass spectrometric data. Journal of Analytical Atomic Spectrometry, 26(12):2508-2518, 2011. doi: 10.1039/c1ja10172b.

J. Taylor. Introduction to error analysis, the study of uncertainties in physical measurements. 1997.

H. Wickham. Tidy data. Journal of statistical software, 59(1):1-23, 2014. doi: 10.1201/9780429200717-7. 